\title{
Cuando los extremos no se encuentran: un análisis de la movilidad social e igualdad de oportunidades en el Perú contemporáneo
}

Des extrêmes qui ne se rejoignent pas : une analyse de la movilité sociale et de l'égalité des chances dans le Pérou d'aujourd'hui

When extremes do not meet: an analysis of social mobility and equal opportunities in present-day Peru

\section{Martín Benavides}

\section{CpenEdition}

\section{Journals}

Edición electrónica

URL: http://journals.openedition.org/bifea/6600

DOI: $10.4000 /$ bifea. 6600

ISSN: 2076-5827

Editor

Institut Français d'Études Andines

Edición impresa

Fecha de publicación: 1 diciembre 2002

Paginación: 473-494

ISSN: 0303-7495

Referencia electrónica

Martín Benavides, «Cuando los extremos no se encuentran: un análisis de la movilidad social e igualdad de oportunidades en el Perú contemporáneo », Bulletin de I'Institut français d'études andines [En línea], 31 (3) | 2002, Publicado el 08 diciembre 2002, consultado el 08 diciembre 2020. URL : http:// journals.openedition.org/bifea/6600; DOl : https://doi.org/10.4000/bifea.6600

\section{cc) (†) $\ominus$}

Les contenus du Bulletin de l'Institut français d'études andines sont mis à disposition selon les termes de la licence Creative Commons Attribution - Pas d'Utilisation Commerciale - Pas de Modification 4.0 International. 


\title{
CUANDO LOS EXTREMOS NO SE ENCUENTRAN: UN ANÁLISIS DE LA MOVILIDAD SOCIAL E IGUALDAD DE OPORTUNIDADES EN EL PERÚ CONTEMPORÁNEO
}

\author{
Martín BENAVIDES*
}

\section{Resumen}

Este trabajo analiza la movilidad social y la igualdad de oportunidades en el Perú contemporáneo. Usando un esquema empírico de clases, se aplican modelos logarítmicos lineales y multiplicativos a una muestra de trabajadores urbanos. Se concluye que los procesos de movilidad relativa entre grupos y el mejoramiento de la igualdad de oportunidades han sido neutralizados tanto por las tendencias negativas de crecimiento como por la falta de cambios importantes en las relaciones económicas y culturales. La estructura de clases resultante en el Perú es una que combina importantes procesos de movilidad social de corta distancia con una limitada movilidad relativa entre clases socialmente distantes. De esa manera, podemos decir que en el Perú se ha producido un "ensanchamiento" de las clases medias, el cual coincide con un comportamiento casi estamental entre los extremos de su estructura social.

Palabras claves: Movilidad social, igualdad de oportunidades, Perú urbano.

\section{DES EXTRÊMES QUI NE SE REJOIGNENT PAS : UNE ANALYSE DE LA MOVILITÉ SOCIALE ET DE L'ÉGALITÉ DES CHANCES DANS LE PÉROU D'AUJOURD'HUI}

\section{Résumé}

Cet article analyse la mobilité sociale et l'égalité des chances au Pérou. Un schéma empirique des classes est d'abord présenté, puis des modèles logarithmiques linéaires et multiplicatifs sont appliqués à un échantillon de travailleurs urbains. Ceci nous a permis de constater que les processus de mobilité relative entre groupes et l'amélioration de l'égalité des chances ont été neutralisés tant par la croissance économique négative que par l'absence de changements importants dans les relations économiques et culturelles. La structure de classes qui en résulte aujourd'hui au Pérou implique donc d'importants processus de mobilité sociale de courte distance et une mobilité relative limitée entre classes socialement éloignées. En ce sens, on peut dire qu'un "élargissement" des classes moyennes a eu lieu et, qu' au même moment, les

*GRADE, Av. del Ejército, 1870 - San Isidro, Lima, Perú. E-mail: mbenavides@ grade.org.pe 
classes socialement éloignées formant partie de la structure sociale péruvienne ont continué à se polariser.

Mots clés : Mobilité sociale, égalité des chances, Pérou urbain.

\title{
WHEN EXTREMES DO NOT MEET: AN ANALYSIS OF SOCIAL MOBILITY AND EQUAL OPPORTUNITIES IN PRESENT-DAY PERU
}

\begin{abstract}
This paper analyses the current trends and patterns of social mobility and equality of opportunity in Peru. After proposing a class schema, we apply log-multiplicative and log linear models to a new data set of urban workers. We conclude that processes of relative mobility and equality of opportunity have been neutralised over time by the negative economic growth rates, as well as by a lack of change in economic and cultural relations. The resulting class structure in present-day Peru is one that combines significant "short distance" mobility with a lack of relative mobility between socially distant classes. Peruvian medium classes are then broadening at the same time than a huge and enduring polarization between socially-distant classes is still reproducing in the social structure.
\end{abstract}

Keys words: Social Mobility, Equality of Oportunity, Urban Peru.

\section{INTRODUCCIÓN}

En la segunda parte del siglo XX, la sociedad peruana ha experimentado importantes cambios. Uno de los motivos principales de dichos cambios han sido los procesos de modernización expresados, entre otras cosas, por las migraciones, la expansión de la educación y la diferenciación ocupacional en los mercados de trabajo. Al mismo tiempo, varios estudios han documentado ya la forma cómo el rostro social del país se ha modificado, por ejemplo a partir del surgimiento de nuevos actores sociales y nuevos espacios urbanos (Franco, 1991).

Sobre esto último no vamos a discutir, aunque uno podría empezar a preguntarse por los datos empíricos que sustentan dichas argumentaciones. En todo caso, hay estudios que cuestionan la idea de los nuevos actores sociales o de la constitución de lo que se ha llamado la "plebe urbana" (Pásara et al., 1991). Este artículo tiene, sin embargo, otro interés. Es nuestra preocupación el responder a la pregunta de la naturaleza de dichos cambios estructurales, específicamente nos interesa saber si se puede decir también que dichos procesos de modernización generaron igualdad de oportunidades. Nos preocupa el tema porque hay muchos trabajos que han ido más allá de las descripciones anteriormente planteadas y han extendido la visión de los cambios a temas un poco más complejos como el de la movilidad social y la circulación y reemplazo de élites (1).

(1) Nos referimos en concreto a la idea de que la migración habría generado procesos importantes de movilidad social para los nuevos pobladores urbanos. 
Sin dejar de reconocer la importancia de algunos de los cambios anteriormente mencionados, en este artículo queremos distanciarnos un tanto de lo que se podría llamar una visión exageradamente optimista sobre los efectos de los cambios estructurales experimentados en las últimas décadas. En ese sentido se discutirán argumentos de por qué las oportunidades sociales no han cambiado drásticamente al menos en lo que concierne a grupos que se ubican en los extremos de la estructura social, y se planteará un modelo de estructura social peruana, el cual señala precisamente la manera cómo en nuestra sociedad se confunden dos procesos de cambios: uno de cambios dinámicos importantes en lo que se podría llamar las franjas intermedias y bajas de nuestra estructura social y otro de comportamiento más bien estamentales en los extremos de dicha estructura.

Para ello empezaremos discutiendo de manera sucinta la noción de igualdad de oportunidades y sus vínculos con el tema de la movilidad social expresada en este caso a partir de cambios en la estructura ocupacional. Luego, discutiremos argumentos de por qué la igualdad de oportunidades no ha cambiado drásticamente en el Perú, para finalmente presentar nuestros datos empíricos y resultados.

\section{LA MOVILIDAD SOCIAL COMO REFLEJO DE IGUALDAD DE OPORTUNIDADES}

La movilidad social es aproximada en este caso a partir de los cambios en las posiciones que los trabajadores peruanos ocupan en la estructura ocupacional en relación con la que ocupaban sus padres. A dicha posiciones les llamaremos clases, en el sentido de posiciones definidas por las relaciones de empleo en los mercados laborales y las unidades de producción en los distintos sectores de las economías nacionales (Goldthorpe, 2000). El análisis de movilidad examinará entonces los procesos por los cuales los individuos y las familias son distribuidos y redistribuidos entre las posiciones de clase a través del tiempo. En ese sentido, se puede decir también que dará información sobre el grado de estabilidad o cambio que las oportunidades —en este caso en el mercado laboral — tienen a lo largo del tiempo.

Un análisis del proceso de movilidad de clases permitirá sacar conclusiones acerca de una determinada estructura de clases. ¿Cuán estable es a lo largo del tiempo? ¿Cómo se distribuyen las oportunidades entre las clases? ¿Cuánta reproducción o sustitución de la élite existe?

Por otro lado, se logrará una sociedad con igualdad de oportunidades si se nivelan las circunstancias sociales que no permiten que todos estén en las mismas condiciones de "juego en el campo". Así, la definición de estas circunstancias sociales se vuelve sumamente importante para la definición de la igualdad de oportunidades. ¿Qué características particulares de la historia del individuo deberían ser niveladas? Según la teoría ello debería ocurrir con las circunstancias de los individuos por las cuales no deberían ser considerados responsables y que afectan su capacidad de logro o de acceso a ciertas ventajas (Roemer, 1998).

Un análisis de movilidad revelará únicamente la manera cómo las diferencias en la clase de origen (ocupaciones de los padres) generan o no generan diferencias en la 
clase de destino (ocupaciones de los hijos), por lo que las circunstancias sociales dentro de este marco sólo se limitan a los orígenes de clase desiguales. Es decir, habrá igualdad de oportunidades si el logro ocupacional (clase de destino) no está relacionado con los antecedentes sociales (clase de origen).

Sin embargo, es importante notar que dicho análisis puede ser criticado porque, aparte de la clase de origen, no aborda otros elementos que están más allá del control de los individuos y que también afectan las oportunidades de vida del individuo.

Tal es, por ejemplo, la crítica que hace John Rawls ( 1971) a la idea liberal de la igualdad de oportunidades. Según él, existen otras desigualdades que no están siendo tomadas en cuenta por esta propuesta liberal; por ejemplo, las disparidades físicas o biológicas, como los talentos naturales. De acuerdo con esta idea, se puede tener sociedades con una "movilidad perfecta" que siguen siendo desiguales porque algunos individuos tienen mejores habilidades naturales que otros.

Por lo tanto, la versión de igualdad de oportunidades que se analizará aquí estará limitada a un solo conjunto de circunstancias particulares - aquéllas derivadas de las desigualdades de clase- - Debido a que la clase de origen podría estar relacionada con otros factores (habilidad y esfuerzo) que pueden influir en el logro ocupacional, una asociación entre clase de origen y clase de destino no descarta la igualdad de oportunidades, tal como se le define aquí.

Dadas las restricciones empíricas que tengo para este análisis, a fin de demostrar la igualdad de oportunidades, será necesario asumir entonces que las correlaciones entre clase de origen y habilidad y clase de origen y esfuerzo son mínimas, de manera que una correlación pequeña o inexistente entre clase de origen y clase de destino pueda indicar igualdad de oportunidades. El efecto independiente de la habilidad natural y del esfuerzo sobre la clase de destino no afectará la igualdad de oportunidades entendida dentro de este marco.

Una vez definida la vinculación entre posición en el mercado laboral, movilidad social e igualdad de oportunidades, procederemos a explicar por qué los procesos de modernización podrían no haber transformado la igualdad de oportunidades.

\section{MODERNIZACIÓN Y MOVILIDAD SOCIAL}

En la literatura comparada, el proceso de modernización ha sido extensamente utilizado para entender el impacto que los cambios económicos y culturales tienen sobre la estructura social, especialmente sobre la distribución relativa de oportunidades.

El argumento básico de la llamada perspectiva liberal es que el proceso de modernización transforma la asignación de los individuos dentro de la división del trabajo. Según dichos enfoques, los cambios tecnológicos y los procesos racionales de selección no dan cabida a la adscripción como criterio de asignación. Más bien, el criterio es el logro. La demanda por más trabajadores calificados también fomentaría la expansión de los sistemas educacionales y aumentaría el acceso a la educación para individuos de distintas procedencias. Desde esta perspectiva, entonces, la selección meritocrática de individuos es la base de la debilidad de la asociación entre las clases 
de origen y de destino (2). El resultado, según este enfoque liberal, serán sociedades menos estratificadas, ya que las clases no desempeñarán un papel importante en la determinación de las posibilidades que tengan los individuos en la vida.

Argumentos similares fueron desarrollados en Latinoamérica. Uno de los teóricos más importantes fue Germani (1981), quien sostuvo que los procesos de movilidad fomentan la integración social a través de la expansión de la "fluidez social" en la estructura de clases. Según él:

"Bajo el impacto acumulativo de algunos de los procesos típicos del desarrollo económico y la modernización social, los factores contextuales que hicieron de la movilidad un elemento disociador o neutralizaron sus consecuencias integrativas en una etapa más temprana tienden a ser reemplazados por las condiciones opuestas. Estos procesos incluyen cambios en el perfil de estratificación - expansión de los estratos intermedios y una resultante 'movilidad estructural' -; una movilidad adicional generada por los diferenciales demográficos; mayor fluidez ocasionada por la movilidad de intercambio generada por una más amplia aplicación de los criterios de logro; una continua transferencia de símbolos de condición social de arriba hacia abajo, a través de una mayor participación en los patrones de consumo, estilos de vida o movilidad superiores." (Germani, 1981: 183, traducción propia)

Por lo tanto, desde esta perspectiva, se afirma que debido a la expansión de la educación, el cambio en el proceso de asignación de individuos al mercado laboral, el crecimiento económico y la urbanización, habrá mayor fluidez entre generaciones. Es decir, no sólo aumentarán las tasas de movilidad absoluta, sino también las tasas de movilidad relativa - aquéllas relacionadas con la circulación de las clases-. Lo mismo ha sido afirmado por estudiosos del caso peruano.

Becker (1983), por ejemplo, pone mayor énfasis en el impacto a corto plazo de la industrialización. Para él, existen procesos de movilidad ascendente como resultado de las nuevas oportunidades creadas por el desarrollo de la industria minera. Según Becker (1983):

“... la tendencia general de la evidencia es que la industria minera, por varios años, ha representado una importante vía de movilidad social ascendente hacia la élite burguesa nacional, por lo menos, para unos cuantos afortunados de los estratos intermedios de una sociedad que, en otros ámbitos, no es considerada como un ejemplo de igualdad de oportunidades... Puede que el ascenso a la alta gerencia no esté exento de nepotismo y clientelismo, pero puede lograrse demostrando competencia profesional." (p. 248, traducción propia)

Por otro lado, también se desarrollaron argumentos sobre los impactos positivos de la migración en la estructura social. El proceso intensivo de migración en la región latinoamericana y especialmente en el Perú, llevó también a algunos teóricos a extender

(2) Un resumen de diferentes otros autores relacionados con esta perspectiva se puede encontrar en Goldthorpe, 2000. 
esa visión optimista y a hablar de procesos potenciales de movilidad social para los hijos de trabajadores rurales. Evaluaciones positivas sobre las motivaciones subjetivas para migrar fueron trasladadas hacia una visión optimista de las trayectorias personales de dichos individuos en el mercado laboral, sin considerar limitaciones estructurales. Incluso algunos llegaron a plantear que esos grupos podrían llegar a formular una nueva burguesía y una nueva generación de profesionales.

Existen, sin embargo, dos razones por las cuales la modernización tiene efectos limitados sobre la estructura social, específicamente sobre la movilidad relativa de clases. En primer lugar, diversas investigaciones han mostrado que el "motor exógeno" de los cambios estructurales (industrialización, crecimiento económico) tiene efectos limitados en el tiempo, debido a que su comportamiento no es constante sino más bien errático. Por ello, tal como Lipset \& Zetterberg (1959) señalan, sus efectos sólo se dan luego de una fase inicial de industrialización o crecimiento, pero luego desaparecen. Los impactos iniciales pueden deberse al hecho de que la expansión fuerza la movilidad al crear mayores puestos de trabajo.

En el Perú, dichas fuerzas exógenas no han sido constantes en el tiempo, tal como podemos observar en el siguiente cuadro, y por lo tanto, no habría razón para esperar cambios crecientes en la igualdad de oportunidades, más allá de aquellos "shocks" iniciales.

Cuadro 1 - Ratios de crecimiento en el sector manufacturero (Seahan, 1999).

\begin{tabular}{|l|cc|}
\hline & 70 's & 80 's \\
\hline Manufactura & 3,3 & $-1,9$ \\
PBI & 3,7 & $-1,2$ \\
\hline
\end{tabular}

La segunda razón por la cual los procesos de modernización no necesariamente modifican igualdad de oportunidades tiene que ver con el hecho de que se puede tener modernización sin alterar profundamente las relaciones sociales en la economía y las relaciones culturales. No sólo es necesario cambiar los marcos institucionales a fin de aumentar la movilidad social, lo que también hay que cambiar es la distribución relativa de las nuevas ventajas generadas por estos cambios institucionales.

Tal como lo muestra la investigación comparada, los grupos en el poder son capaces de beneficiarse con las oportunidades generadas por las transformaciones económicas. Desde este punto de vista, los estudiosos del proceso de transición de las sociedades comunistas a las de mercado, o de las sociedades prerevolucionarias a las posrevolucionarias, aclaran el tema de la reproducción de las ventajas de clase o los procesos de supervivencia de la élite en las distintas fases de las transiciones económicas. Así, los denominados "enfoques de la supervivencia de la élite" estudian de qué manera los ex miembros del partido comunista que durante el comunismo tuvieron puestos privilegiados, pudieron mantener sus puestos aún en la etapa del poscomunismo. Estos 
enfoques resaltan dos mecanismos diferentes: las estrategias de conversión del poder y las diferencias relativas del capital humano. Con respecto a las estrategias de conversión del poder, Rona-Tas (1994) y otros han subrayado la idea que la posición privilegiada de los cuadros políticos no necesariamente cambia después de la transformación. El enfoque de la conversión del poder sostiene que "el poder acumulado durante el socialismo de estado se convierte en activos de alto valor en la economía de mercado" (Rona-Tas, 1994). Estos cuadros utilizan sus redes personales para tener acceso a información valiosa dentro del sistema.

Los análisis del proceso de transición a las sociedades postcomunistas también utilizan un argumento relacionado con el capital humano. En la nueva situación de mercado, a los que tienen mayor educación les irá mejor que a los que tienen un menor nivel de educación. El cuadro tecnocrático que se desarrolló durante el socialismo puede lograr mantener su posición después de la erosión del sistema. Estas ideas son parecidas a aquellas que discutían la transformación social en otras partes del mundo. En sus análisis del impacto de la transformación boliviana en la estructura de clases, Kelley \& Klein (1981) sostienen que la desigualdad volvió a surgir debido a que la revolución no pudo eliminar las diferencias previas en capital humano y redes sociales.

En el Perú, un argumento que ha sido ampliamente discutido en esa línea es el de la modernización tradicionalista. Se sostiene que las distintas fases del proceso de modernización han sido dirigidas por las élites tradicionales, las cuales lograron transformarse y adaptarse a las nuevas situaciones. Según el concepto de la modernización tradicionalista, la estructura social peruana recibió elementos capitalistas, pero sin cambiar la estratificación social. Así, es la clase dirigente tradicional la que habría modernizado la sociedad (Guadalupe, 1991).

En una tónica similar, otros investigadores sostienen que barreras culturales a la movilidad también operan en la estructura de clases peruana. Seguirían manifestándose algunas identidades poderosas, a pesar de una aparente tendencia hacia procesos capitalistas más abiertos. La mayoría de los sectores vinculados a empresas industriales no confíaría así ni en la posibilidad de tener un verdadero mercado (Nugent, 1992).

Dichos argumentos sobre los límites de los procesos de modernización para alterar profundamente la igualdad de oportunidades, nos llevan a plantear las siguientes dos hipótesis de análisis. La primera es que las tasas de movilidad relativa entre clases ocupacionales serán muy similares para diferentes cohortes de edad. La segunda hipótesis es que el patrón de movilidad social y, por lo tanto, de la estructura social peruana, no coincide con uno en el cual las diferencias relativas entre clases socialmente distantes se hayan acortado. Se espera encontrar mucha movilidad pero coincidente con pocos cambios relativos en los grupos socialmente distantes.

\section{DATOS}

Para este estudio, se ha utilizado los datos de la Encuesta de Medición de Niveles de Vida (ENNIV), realizada los años 2000 y 2001 en las zonas urbanas de Perú. La ENNIV es una encuesta que se realiza en diversos países con el auspicio del Banco Mundial, en la cual se recolecta datos sobre diferentes dimensiones del bienestar 
familiar, incluyendo el consumo, empleo, ingreso, ahorro, educación, fertilidad y nutrición. El informante es el jefe de familia, y la encuesta es representativa de los hogares peruanos a nivel nacional.

En el año 2001, se llevó a cabo un complemento de la ENNIV para un proyecto de exclusión social realizado por el Grupo de Análisis para el Desarrollo (GRADE). Esta última encuesta se aplicó a una fracción significativa de miembros de hogares urbanos, mayores de 18 años de edad, e incluyó variables que fueron omitidas en la versión del año 2000, tales como ocupación del padre, raza y estilo de vida.

En esta muestra, se encuestó a 5700 individuos. Si bien el módulo sólo abarcó $70 \%$ de la muestra original del año 2001, no hubo diferencias significativas en las principales características entre la muestra urbana y la población total. El $30 \%$ de los casos que se perdieron no parece haber generado mayores problemas (Torero et al., 2002). Además, la distribución ocupacional de los trabajadores urbanos de sexo masculino entre 25 y 65 años de edad fue muy similar en los dos segmentos de la encuesta.

El interés de este artículo es la movilidad ocupacional de los hombres. A fin de evitar el problema de la mortalidad, la muestra se circunscribió a individuos de menos de 65 años de edad. Asimismo, la muestra se limitó a los trabajadores de más de 25 años de edad. Así, tratamos de evitar el problema de aquéllos que aún no han obtenido el título universitario y no han iniciado una carrera ocupacional. Debido a que la ocupación es indefinida para aquéllos que no están trabajando, también se excluyó de la muestra a los desempleados. La muestra analítica final de individuos de sexo masculino de áreas urbanas, con información sobre clase de origen y de destino y entre 25 y 65 años de edad comprendió a 1700 individuos.

\section{VARIABLES}

\section{1. Clase}

Dentro del contex to de la estructura de clases, esta investigación seguirá la lógica del esquema de clases ocupacionales propuesto por Goldthorpe (2000) (3). Esta lógica empezó con la introducción de distinciones básicas entre los que compran el trabajo de otros, los que no compran el trabajo de otros pero no venden el propio, y los que venden su trabajo a otros. Debido a que la mayor parte de la fuerza laboral de los países industrializados terminará en la tercera categoría —empleados—, la diferenciación básica de este enfoque es aquella que se da entre tipos de empleados (Goldthorpe, 2000). Por ello, la atención se centra en la naturaleza de los contratos de empleo. Según Eriksson \& Goldthorpe (1992), hay dos formas básicas de relaciones de empleo: el contrato de trabajo y la relación de servicio:

(3) Esta es una decisión conceptual. Dada la naturaleza relacional del problema de la igualdad de oportunidades, hemos optado por una aproximación empírica que trabaje con categorías y no con índices continuos, los cuales además asumen un comportamiento lineal que no necesariamente corresponde con la estructura social peruana. Esta discusión puede encontrarse en Hout (1982). 
"Las relaciones de empleo reguladas por un contrato de trabajo conllevan un intercambio específico de dinero por esfuerzo por un plazo relativamente corto. Los empleados proporcionan cantidades de trabajo más o menos discretas, bajo la supervisión del empleador o del agente del empleador, a cambio de un salario, que se calcula 'a destajo' o 'por tiempo trabajado'. En contraste, las relaciones de empleo dentro de un contexto burocrático involucran un intercambio a más largo plazo y, generalmente, más difuso. Los empleados brindan un servicio a la organización que los emplea, a cambio de una 'compensación', que no sólo adopta la forma de una recompensa por el trabajo realizado a través de un sueldo y diversos emolumentos, sino que también comprende importantes elementos esperados — como aumentos de sueldo en una escala establecida, garantía de seguridad tanto en el empleo como a través de los derechos de pensión después de la jubilación y, sobre todo, oportunidades profesionales bien definidas-." (p. 41, traducción propia)

Además de estas dos formas de relación de empleo, los autores consideran también las formas intermedias, aquellas compuestas por relaciones que tienen características de las dos formas anteriormente planteadas.

No obstante, el esquema empírico de clases, desarrollado para los países industrializados de acuerdo con esta lógica analítica, tiene algunas características específicas que hacen que su aplicación en el Perú resulte problemática. Me estoy refiriendo aquí a la premisa principal del esquema de Goldthorpe, es decir, que las relaciones entre empleados son bastante estables y que es posible capturarlas analizando las distintas posibilidades contractuales.

El principal problema con el caso peruano tiene que ver con la gran cantidad de empleados informales que no pueden ser considerados como parte de un servicio, relación intermedia o relación laboral (Castells \& Portes, 1989). La relación informal es, básicamente, una relación sin contrato. Los empleados son, principalmente, familiares que trabajan sin remuneración en pequeños negocios; por ejemplo, los vendedores ambulantes.

Hay dos características que diferencian a las relaciones de empleo de los vendedores ambulantes de las relaciones reflejadas en un contrato laboral: la manera cómo se rigen y la volatilidad. En la relación de empleo informal, están presentes los acuerdos familiares o a corto plazo entre empleador y empleado. La volatilidad se ve reflejada en la ausencia de un acuerdo enmarcado en el tiempo, referido a la duración de la relación.

Por lo tanto, además de las tres principales fuentes de relaciones de empleo, he decidido crear otra: la forma casual de relación. Los vendedores ambulantes o los autoempleados no calificados que no tienen personas que trabajen para ellos estarán clasificados en esta categoría. Los auto-empleados casuales, como los vendedores ambulantes, se diferencian de otros auto-empleados más tradicionales, como los artesanos, técnicos, etc. La diferencia radica, mayormente, en la volatilidad y la falta de acumulación de los vendedores ambulantes. En el caso de la volatilidad, sus actividades 
se pueden relacionar con distintas formas de negocio. No necesariamente son las mismas a corto plazo. Un día pueden vender banderas; al día siguiente, comida. En el caso de la acumulación, se puede decir que tales actividades son, mayormente, de supervivencia. Por esta razón, se ha clasificado a todas las actividades de auto-empleo no calificado y que cuentan con trabajadores, "la pequeña burguesía casual”, junto con los trabajadores casuales.

Al mismo tiempo, he agregado una diferenciación entre dos formas de pequeña burguesía. La pequeña burguesía A está conformada por pequeños empleadores, mientras que la pequeña burguesía B está conformada por autoempleados calificados, y vendedores ambulantes con trabajadores. La diferencia entre las dos radica en la situación más estable de los pequeños empleadores en relación con la fase de transición de la pequeña burguesía $B$.

Por otro lado, mientras el esquema de clases de Goldthorpe considera a los técnicos de nivel inferior y a los trabajadores semi-calificados como una sola clase, las diferencias en el prestigio asociado con estos dos grupos en el Perú nos llevan a considerarlos como dos clases separadas. En esta diferenciación, la cantidad de educación invertida por el primer grupo en comparación con el segundo desempeña un papel importante.

Siguiendo dicha lógica se ha validado un esquema de clases usando tanto una validación de constructo como de criterio (Benavides, 2002). Dicho esquema de clases puede observarse en la sección de resultados.

\section{2. Cohorte}

Para el análisis de las tendencias, se han definido tres cohortes distintas. La primera cohorte está conformada por individuos entre 50 y 65 años de edad al momento de la encuesta. Estas personas se incorporaron en la fuerza laboral entre 1960 y 1975 , período en el cual se llevó a cabo el proceso de industrialización y reformas de la propiedad más importantes en el país. La segunda cohorte está conformada por individuos entre 35 y 49 años de edad al momento de la encuesta, y que son los que empezaron sus carreras laborales después del proceso reformista militar, entre 1975 y 1990, un período de tasas negativas de industrialización y de crecimiento. Finalmente, la última cohorte está compuesta por aquéllos que iniciaron sus carreras durante o después de los procesos de reforma de mercados en la década de 1990.

\section{MÉTODOS ESTADÍSTICOS}

Para analizar las tendencias en cuanto a la igualdad de oportunidades, se calculará las tasas relativas de movilidad en base a dos modelos diferentes: el logarítmico lineal y el logarítmico multiplicativo.

El primer modelo - el logarítmico lineal- es el modelo de fluidez social constante (Eriksson \& Goldthorpe, 1992). Según este modelo, el nivel de asociación entre clase de origen y clase de destino - y por lo tanto de igualdad de oportunidadeses constante para las tres cohortes 


$$
\log F_{i j k}=\mu+\lambda_{i}^{O}+\lambda_{j}^{D}+\lambda_{k}^{C+} \lambda_{i k}^{O C}+\lambda_{j k}^{D C}+\lambda_{i j}^{O D}
$$

donde $\mathrm{f}_{\mathrm{ijk}}$ es la frecuencia esperada en la celda ijk en la tabla tri-direccional que contiene clase de origen $(\mathrm{O})$, clase de destino (D) y cohorte $(\mathrm{C}) ; \mu$ es un factor de escala; O, D y $\mathrm{C}$ son los principales efectos de la distribución de los individuos a través de los orígenes, destinos y cohortes, respectivamente; y los otros parámetros representan los efectos de las asociaciones bi-direccionales en la tabla (4).

A fin de probar si han ocurrido ciertos cambios entre las cohortes y si dichos cambios siguen una dirección específica, se usará el modelo logarítmico multiplicativo "unidiff" (Eriksson \& Goldthorpe, 1992). Este modelo sustituye el parámetro de asociación entre origen y destino en el modelo de fluidez social constante con un parámetro $\beta_{\mathrm{k}} X_{\mathrm{ijj}}$, donde $\mathrm{X}_{\mathrm{ij}}$ representa el patrón general de asociación entre origen $\mathrm{y}$ destino y $\beta_{\mathrm{k}}$ es la fuerza relativa de la asociación, que es específica para una cohorte (Eriksson \& Goldthorpe, 1992).

$$
\log F_{i j k}=\mu+\lambda_{i}^{O}+\lambda_{j}^{D}+\lambda_{k}^{C}+\lambda_{i k}^{O C}+\lambda_{j k}^{D C}+\beta_{k} \mathrm{X}
$$

En segundo lugar, para el análisis del patrón nacional de movilidad social, usaré el modelo logarítmico lineal topológico. Este modelo permite que todos los conjuntos de asociaciones en una tabla de movilidad pueden ser explicados por parámetros de "nivel". Los conjuntos de celdas (origen y destino) que, de acuerdo con cierta hipótesis, comparten similares oportunidades relativas de movilidad, tendrán el mismo nivel. Los niveles están numerados de la densidad más alta (mayor concentración de casos en la celda) a la más baja, de acuerdo con los efectos marginales. Formalmente, el modelo es como sigue:

$$
\log _{i j}=U+\lambda_{i} O+\lambda_{j} S+\lambda_{k} D
$$

donde $\mathrm{U}$ es un factor de escala, $\mathrm{O}$ y $\mathrm{S}$ son los parámetros horizontales y verticales, y D es el parámetro de densidad que reemplaza al término de interacción.

\section{RESULTADOS}

Para poder hacer comparaciones entre zonas de la estructura ocupacional en vez de clases ocupacionales por sí mismas, hemos divido la estructura de clases en tres zonas diferentes. La zona 1 será la zona de mayor prestigio, la zona 3 la de menos prestigio y la zona 2 la de las clases intermedias. Siguiendo asociaciones entre clases ocupacionales, ingresos y educación, los profesionales y administradores de alto y bajo nivel son considerados dentro de la zona 1 , mientras los obreros, trabajadores no calificados,

(4) Para este modelo, y dado el número limitado de casos, el esquema de nueve clases ha sido reducido a uno de seis. Esto ha sido realizado tomando en cuenta ingresos similares y condiciones similares. 
trabajadores casuales y rurales están en la zona 3. Tal como se observa en el cuadro siguiente, las clases restantes están en la zona 2.

Cuadro 2 - Clases ocupacionales según años de educación e ingresos.

\begin{tabular}{|c|c|c|c|}
\hline Clases & $\begin{array}{l}\text { Años de } \\
\text { educ. }\end{array}$ & $\begin{array}{c}\text { Ingr. } \\
\text { mensual } \\
\text { (en soles } \\
\text { de Lima) }\end{array}$ & Zona \\
\hline I. Profesionales y administradores de nivel superior & 15,65 & 1989 & 1 \\
\hline II. Profesionales y administradores de nivel inferior & 15,57 & 1277 & 1 \\
\hline IIIa. Pequeña burguesía A & 10,89 & 1270 & 2 \\
\hline IIIb. Pequeña burguesía B & 9,43 & 735 & 2 \\
\hline IV. Empleados & 12,57 & 1032 & 2 \\
\hline V. Técnicos de nivel inferior y trabajadores calificados & 12,21 & 941 & 2 \\
\hline VI. Trabajadores obreros semi-calificados & 9,84 & 748 & 3 \\
\hline VII. Trabajadores casuales y no calificados & 9,23 & 622 & 3 \\
\hline VIII. Trabajadores rurales & 7,79 & 631 & 3 \\
\hline
\end{tabular}

Con los niveles definidos de esa manera, se pueden calcular entonces procesos de movilidad ascendente, descendente, y horizontal. El primero - la movilidad ascendente- será el porcentaje de los individuos móviles (aquellos cuya clase ocupacional de destino es diferente de las de sus padres) que se mueven desde la zona 3 a la 2, o desde la zona 2 a la 1. La movilidad horizontal será definida por el porcentaje de aquellos individuos móviles que se quedan en el mismo nivel, mientras que la movilidad descendente será definida por el porcentaje de individuos que se mueven desde la zona 1 a la 2, o desde la zona 2 a la 3.

Los cálculos muestran que la movilidad ascendente comprende el $40 \%$ de la movilidad total, mientras que la movilidad horizontal el $35 \%$ y la movilidad descendente el $25 \%$. Son necesarias dos precisiones en cuanto a estos datos. Lo primero es resaltar que no toda la movilidad ha sido ascendente tal como muchas veces se asume. La movilidad descendente y la movilidad horizontal comprenden buena parte de la movilidad total. Lo segundo es que la movilidad analizada de esta forma no controla los cambios estructurales, tales como los cambios demográficos, la expansión económica. 
Las tasas relativas son una medida más exacta para el problema de la igualdad de oportunidades pues están controladas por dichos procesos. Las tasas de movilidad relativas se refieren al "régimen de movilidad endógena" (Hauser, 1978) o "patrón de fluidez social" (Eriksson \& Goldthorpe, 1992). A dicha movilidad relativa también se le denomina medida de la desigualdad de oportunidades, y es el punto principal de este análisis. La siguiente discusión analiza los cambios en esas tasas relativas.

\section{1. A pesar de la modernización las tasas relativas se mantienen constantes}

El siguiente cuadro examina las tendencias de las tasas de movilidad relativa para las diferentes cohortes. En primer lugar, podemos ver que el modelo de fluidez constante encaja con los datos. Según este modelo, la asociación neta (de la movilidad estructural) entre origen y destino es la misma para todas las cohortes. Existen importantes elementos de las tasas de movilidad relativa que son comunes para las tres cohortes. Sin embargo, este modelo aún puede ser mejorado con el "unidiff”. Elíndice de discrepancias es $7 \%$, lo cual significa que $7 \%$ de las asociaciones en este modelo no son explicadas satisfactoriamente por el modelo de fluidez constante. Con el modelo "unidiff", veremos si es posible determinar si las pequeñas diferencias — que no son reflejadas por el modelo de fluidez constante- muestran alguna tendencia en particular.

Cuadro 3 - Tendencias en las tasas de movilidad relativa a través de las cohortes.

\begin{tabular}{|c|c|c|c|c|c|}
\hline \multicolumn{2}{|c|}{$\begin{array}{c}\text { Ratio absoluto } \\
\text { de movilidad }\end{array}$} & \multicolumn{2}{|c|}{ "Deviance" Df } & \multirow{2}{*}{$\begin{array}{c}\begin{array}{l}\text { Índice de } \\
\text { discrepancia }\end{array} \\
7 \%\end{array}$} & \multirow[t]{2}{*}{$\begin{array}{l}\text { Parámetro de } \\
\text { asociación }\end{array}$} \\
\hline Fluidez constante & & 56 & 50 & & \\
\hline Unidiff & & 52 & 48 & $5 \%$ & \\
\hline Cohorte Joven & $70 \%$ & & & & $1,43^{*}$ \\
\hline Cohorte Intermedia & $72 \%$ & & & & $1,31 *$ \\
\hline Cohorte Antigua & $76 \%$ & & & & 1 (fijado a 1$)$ \\
\hline
\end{tabular}

$* \mathrm{p}<.10$

El “unidiff” mejora el ajuste de los modelos, pero sin alcanzar significancia según niveles convencionales (la desviación es 4 por cada 2 grados de libertad perdidos). Al analizar los parámetros $\mathrm{B}$, se puede observar que los niveles de fluidez han disminuido para la cohorte intermedia (con un nivel de significancia de 10\%), y parecen permanecer constantes para la cohorte más joven. No obstante, los resultados correspondientes a la cohorte más joven deben ser tomados con cierta reserva, debido a la composición de dicha cohorte (donde algunos individuos tal vez no se encuentren aún en su nivel de "madurez ocupacional", por lo que podría haber un efecto de edad). En todo caso, el cambio en las tasas de movilidad que experimenta la cohorte de mayor edad se nivela para la cohorte siguiente. 
Esto es lo que los resultados sugieren para el Perú. Como en la mayoría de los países, las cohortes en el Perú comparten importantes elementos en sus tasas de movilidad relativa. Como ocurrió en otros países, el impacto positivo de un crecimiento económico temprano fue neutralizado por una persistente desigualdad y no habría llegado a impactar tan significativamente la estructura social. A diferencia de otros países, las tasas de movilidad relativa para la cohorte intermedia se redujeron debido al impacto de la desindustrialización y el crecimiento negativo y a las grandes desigualdades acumuladas en otros activos. En dichos escenarios, los recursos tipo la educación o las redes sociales — relacionadas ambas con la clase de origen — adquieren nueva importancia al aumentar la competencia por un número limitado de puestos importantes.

\section{2. El patrón de movilidad social en el Perú}

En esta sección, deseo establecer un modelo de movilidad para la sociedad peruana, utilizando el esquema de clases de nueve categorías definido anteriormente, así como algunos argumentos acerca de la estructura social peruana. La cultura popular en la sociedad peruana tiene ideas muy claras acerca de la rigidez de la estructura de clases, especialmente de qué manera las diferencias en las posiciones de clase han estado históricamente vinculadas y estructuradas de acuerdo a formas étnicas y raciales de dominación. Dos de esas imágenes son la del "huachafo" y la del "arribista", las cuales califican en términos negativos a los individuos que intentan pasar de posiciones inferiores a posiciones superiores en la estructura de clases. Mientras la imagen del "arribista" está más claramente relacionada con el intento de moverse en la jerarquía de posiciones, la imagen del "huachafo" tiene mayor carga cultural y descalifica a los individuos pertenecientes a las culturas no dominantes por tratar de ser parte de las clases privilegiadas. Como indica Chaplin (1967), estas imágenes provienen de una sociedad muy rígida, donde la verdadera movilidad es reemplazada por intentos simbólicos de moverse a través de la jerarquía mediante una imitación del comportamiento de las élites. Según Chaplin,

"La movilidad ascendente hacia una condición social de clase media en América Latina ha significado la imitación de los patrones de comportamiento de las élites tradicionales, según la percepción imperfecta de los emuladores, más que la creación de una ética burguesa independiente en base a la cual se podría mirar por encima del hombro a algunos comportamientos de la élite." (p. 223, traducción propia)

O los intentos por desplazarse dentro de la estructura están relacionados con dimensiones no referidas a la posición económica. Según Chaplin (1967)

“... en América Latina, donde la movilidad social basada en el logro económico resulta tan difícil, se recurre ampliamente a la movilidad simbólica... En los pueblos de las provincias, la clase media baja 'se eleva' en sus propios ojos al adoptar una gran cantidad de compadres de clase inferior. Aquéllos que tienen un título universitario se vuelven doctores ante los ojos de los que no han seguido estudios universitarios. Los miembros de mayor edad de la clase superior dicen que el comportamiento huachafo 
(un peruanismo que equivale a cursi u ostentación vulgar pretenciosa) caracteriza a toda la clase media, así como a los nuevos ricos.” (p. 224, traducción propia)

Tomando en cuenta la operación de los procesos de herencia, los procesos de movilidad ascendente y descendente, los siguientes argumentos servirán de base para proponer el modelo peruano de movilidad.

Las clases I y II en nuestro esquema de clases ya definido tienen ventajas relativas en términos de ingreso económico, recursos culturales y sociales (redes), que también se convierten en barreras al acceso a esta clase. Las clases VI (obrera), VII (casual y no calificada) y VIII (agrícola) son las que menos ventajas tienen. Por ello, las clases VI, VII y VIII son las que tienen mayores barreras para intentar ingresar a las clases superiores.

Además de los procesos de herencia esperados en las clases superiores, también hay que tener en cuenta los procesos de herencia específicos en la pequeña burguesía $\mathrm{B}$. A pesar que existe la creencia que esta clase tiene posibilidades de convertirse en la "nueva burguesía” en el Perú (De Soto, 1986), la hipótesis que planteo en este modelo es que, por el contrario, este grupo enfrenta varias barreras para un movimiento ascendente hacia los lugares ventajosos superiores en la estructura de clases. Por esta razón, he modelado para ellos importantes procesos de herencia (IIIb).

También es necesario tomar en cuenta el impacto que tienen los procesos de migración en la estructura social. La posibilidad que tienen los nuevos "habitantes urbanos" de ascender en la estructura social se ve restringida por lo que se ha denominado la "anti-modernidad" de la sociedad peruana, que se refiere al proceso por el cual los artefactos culturales y materiales de la modernización permiten a las clases superiores sentirse más "señores" y tratar a la nueva población urbana como más indígena (Nugent, 1992). Esta tesis contrasta con otras más optimistas. Por ello, el modelo plantea que los hijos de los trabajadores rurales (VIII) que viven en ambientes urbanos son más propensos a seguir trabajando en ocupaciones rurales (cerca a las ciudades) o volverse obreros (VI), trabajadores casuales (VII) o una pequeña burguesía (IIIb). Se plantea la hipótesis que la movilidad de estos individuos es más horizontal o una movilidad a corta distancia hacia la pequeña burguesía $B$, en vez de un proceso de movilidad ascendente hacia las clase profesional.

Al mismo tiempo, se espera que las clases obrera y casual también muestren mecanismos de reproducción. En este caso, el proceso puede entenderse como la combinación de una inmovilidad estructural forzada, resultante de la falta de espacio en la cúspide de la estructura, y barreras al movimiento ascendente, las cuales incluyen la raza/etnicidad y la educación.

También se espera que existan procesos de movilidad descendentes, especialmente aquéllos relacionados con las categorías profesionales inferiores (clase II). La pérdida de ventajas en esta clase durante las últimas generaciones ha sido ampliamente discutida en la literatura sobre el mercado laboral peruano.

El caso de los empleados (IV) también resulta interesante en este sentido. La literatura correspondiente ha puesto mucho énfasis en las afinidades entre esta clase y 
los profesionales y gerentes de los niveles superior e inferior, especialmente durante la primera mitad del siglo XX. Por ejemplo, según Parker (1998),

“... los empleados gozaban de un prestigio del que, indudablemente, carecían los trabajadores manuales. El supuesto desdén hispano hacia el trabajo manual tuvo cierta influencia en la condición social superior del empleado, pero igualmente importantes fueron el paternalismo que caracterizaba a las relaciones empleador-empleado, la tradición de ascenso interno en las firmas comerciales y una serie de costumbres y actitudes que reforzaron la percepción de que los dueños de los negocios y sus empleados pertenecían al mismo mundo social. Más allá de esto, resulta igualmente evidente que la mayoría de los empleados ya fueran prósperos o tuvieran dificultades para salir adelante, vivían en un universo cultural impregnado de los valores e ideales de la élite. En lo que respecta a la vivienda, vestido, alimento y entretenimiento, la clase superior marcaba el ritmo y los 'empleados' lo seguían lo mejor que podían, a veces a costa de grandes sacrificios para distinguirse de la mayoría de peruanos." (p. 63, traducción propia)

No obstante, no tengo un argumento a priori que sostenga que estas afinidades especiales persistirán durante la segunda mitad del siglo. Por el contrario, me inclino a pensar que los empleados tienen los mismos problemas para reproducirse que la clase II. Por ello, no voy a plantear la existencia de vínculos especiales entre las clases I y II y la clase IV.

Siguiendo esta lógica y en base a los argumentos previamente discutidos, tratamos de formalizar la estructura topológica para la estructura de clases peruana. Este modelo debería reflejar las siguientes características típicas del Perú:

- las barreras a la movilidad desde las clases inferiores hacia las clases superiores

- los procesos de herencia característicos de las clases superiores, las clases inferiores, los trabajadores rurales y la pequeña burguesía $B$

- la fluidez entre los niveles intermedios y entre las clases trabajadoras, trabajadores calificados con la pequeña burguesía B

- los procesos de movilidad descendente, especialmente desde las clases II y IV hacia los niveles inferiores.

Este modelo asigna las celdas internas de las tablas de movilidad a una serie de subconjuntos o "regiones", y requiere que todas las celdas en un subconjunto tengan el mismo nivel de asociación neta entre la clase de origen y la clase de destino. Las celdas que, de acuerdo con la teoría, supuestamente deberían tener el nivel más alto de asociación entre clase de origen y clase de destino, o una "alta densidad" entre ellas, están caracterizadas con el número 1. Las celdas con los niveles más bajos de asociación, o baja densidad, tendrán el número 5. Por esta razón, las regiones formadas por celdas con el número 3 son consideradas regiones de alta fluidez.

La estructura se presenta en el cuadro 4, y es el modelo que encaja mejor con las frecuencias observadas de la tabla de movilidad. Este modelo tiene el mejor ajuste con los datos (la desviación es 35 por 60 grados de libertad). 


\section{Cuadro 4 - La estructura topológica peruana.}

\begin{tabular}{|c|c|c|c|c|c|c|c|c|c|}
\hline & & & & Clase & $D e$ & & & & \\
\hline & $I$ & II & IIIa & $I I l b$ & $I V$ & $V$ & $V I$ & VII & VIII \\
\hline$I$ & 1 & 2 & 4 & 4 & 4 & 2 & 4 & 4 & 5 \\
\hline II & 2 & 2 & 4 & 3 & 3 & 3 & 4 & 4 & 5 \\
\hline IIIa & 4 & 3 & 4 & 3 & 4 & 4 & 3 & 3 & 5 \\
\hline$I I I b$ & 5 & 3 & 3 & 1 & 3 & 3 & 1 & 1 & 4 \\
\hline$I V$ & 4 & 3 & 4 & 3 & 3 & 3 & 3 & 3 & 5 \\
\hline$V$ & 4 & 3 & 4 & 4 & 4 & 3 & 3 & 4 & 5 \\
\hline VI & 5 & 4 & 4 & 2 & 4 & 3 & 1 & 2 & 4 \\
\hline VII & 5 & 4 & 5 & 3 & 4 & 4 & 3 & 2 & 4 \\
\hline VIII & 5 & 3 & 3 & 1 & 4 & 3 & 1 & 1 & 1 \\
\hline
\end{tabular}

$\mathrm{I}=$ Profesionales y administradores de nivel superior; II = Profesionales y administradores de nivel inferior; IIIa = Pequeña Burguesía A; IIIb = Pequeña Burgesía B; IV = Empleados; V = Técnicos y trabajadores calificados; VI $=$ Obreros; VII $=$ Trabajadores casuales y no calificados; $\mathrm{VIII}=$ Trabajadores rurales.

Esta estructura revela la importancia de la inmovilidad, particularmente para las celdas de las clases más privilegiadas y de las menos privilegiadas. Esto aparece reflejado en el nivel de alta densidad (1 y 2 en la mayoría de los casos) en la diagonal. Es importante, también, observar el alto nivel de herencia para los trabajadores rurales y la pequeña burguesía B (nivel 1). Una menor herencia se observa en los niveles intermedios, como la pequeña burguesía A (nivel 4), empleados (nivel 3) y técnicos de bajo nivel y trabajadores calificados (nivel 3).

Asimismo, este modelo de movilidad refleja las barreras tanto para el ingreso a las clases más privilegiadas, como para salir de las mismas. El nivel de densidad para estas clases (I y II) disminuye al alejarnos de ellas.

Este modelo también captura los procesos de movilidad descendente desde la clase I hacia la clase $\mathrm{V}$, pero, especialmente, desde la clase II hacia la pequeña burguesía (IIIb), la clase de los empleados (IV) y las clases técnicas de nivel inferior y trabajadores calificados (V). Asimismo, es posible observar un proceso más inusual, pero real, de movilidad descendente desde las clases superiores I y II hacia las clases obrera (VI) y casual (VII). En ambos casos, los niveles de densidad son 4. Este modelo también refleja la situación de la clase de los empleados. Esta clase no muestra una afinidad especial con las clases superiores y, más bien, muestra tendencias muy similares para moverse hacia las distintas clases de la estructura (la mayoría de los niveles de densidad son 3). 
Al mismo tiempo, es interesante observarel patrón de movilidad de los trabajadores que viven en ambientes urbanos, pero que tienen padres con ocupaciones rurales. Estos trabajadores están concentrados en ocupaciones manuales (VI, VII) y también en ocupaciones rurales que pueden estar ubicadas cerca al entorno rural (VIII).

Con este modelo, podemos calcular las oportunidades relativas de movilidad de cualquier par de clases de origen y destino. Tal como lo describe Goldthorpe (1987), esto se calcula de la siguiente manera:

$$
\mathrm{F}_{i k} / F_{i l} \div F_{j k} / F_{j l}=D_{i k} / D_{i l} \div D_{j k} / D_{j l},
$$

donde "los subíndices 1 y j se refieren a las clases de origen; los subíndices k y l, a las clases de destino; F es la frecuencia de las celdas generadas por el modelo; y D es el parámetro para el nivel de interacción al que se asigna una celda" (Goldthorpe, 1987: 59).

A fin de calcular estas oportunidades relativas, es necesario calcular las diferencias de oportunidad entre los niveles. Para ello, es necesario usar la información sobre el cálculo de los parámetros del modelo topológico que ya presentamos anteriormente. El nivel 5 está restringido a 0 , tal como se muestra en el cuadro 5 .

\section{Cuadro 5 - Parámetros del modelo topológico.}

\begin{tabular}{|c|c|}
\hline Nivel & Parámetro \\
\hline 1 & 3,02 \\
2 & 2,65 \\
3 & 2,13 \\
4 & 1,40 \\
5 & 0 \\
\hline
\end{tabular}

Con esta información, es posible determinar las diferencias de oportunidad entre los niveles. Por ejemplo, la diferencia entre los niveles 1 y 2 se convierte en:

$$
e^{(3,02-2,65)}=1,44
$$

Con este método, finalmente podemos calcular todas las diferencias necesarias para calcular las oportunidades relativas, las cuales aparecen en el cuadro 6:

Cuadro 6 - Diferencias entre parámetros de nivel del modelo topológico.

\begin{tabular}{|llllll|}
\hline Niveles & $\mathbf{1}$ & $\mathbf{2}$ & $\mathbf{3}$ & $\mathbf{4}$ & $\mathbf{5}$ \\
\hline 1 & 1 & 1,44 & 2,43 & 5,05 & 20,4 \\
2 & 0,69 & 1 & 1,68 & 3,49 & 14,15 \\
3 & 0,41 & 0,59 & 1 & 2,07 & 8,41 \\
4 & 0,197 & 0,28 & 0,48 & 1 & 4,05 \\
5 & 0,04 & 0,07 & 0,12 & 0,25 & 1 \\
\hline
\end{tabular}


Y, luego, utilizando la fórmula para calcular las oportunidades relativas ,podemos generar las tasas de probabilidad, las cuales aparecen en el cuadro 7.

Cuado 7 - Ratios relativos de movilidad para diferentes clases.

\begin{tabular}{|c|c|c|c|c|c|c|c|c|}
\hline & II & IIIa & IIIb & IV & $\mathbf{V}$ & VI & VII & VIII \\
\hline I & 1,44 & 5,05 & 126 & 10,52 & 3,00 & 126 & 72 & 510 \\
\hline II & & 1,69 & 4,10 & 1,68 & 1,68 & 17,7 & 12,46 & 34,51 \\
\hline IIIa & & & 1,17 & 2,08 & 2,08 & 2,44 & 6,86 & 9,88 \\
\hline IIIJ & & & & 2,43 & 5,06 & 1,45 & 1,69 & 5,05 \\
\hline IV & & & & & 2,08 & 5,08 & 3,57 & 42,69 \\
\hline V & & & & & & 2,44 & 7,39 & 20,51 \\
\hline VI & & & & & & & 2,44 & 5,04 \\
\hline VII & & & & & & & & 3,49 \\
\hline
\end{tabular}

$\mathrm{I}$ = Profesionales y administradores de alto nivel; II = Profesionales y administradores de bajo nivel; IIIa = Pequeña Burguesía A; IIIb = Pequeña Burgesía B; IV = Empleados; V = Técnicos de nivel inferior y trabajadores calificados; VI = Obreros; VII = Trabajadores casuales y no calificados; VIII $=$ Tabajadores rurales.

La desigualdad de oportunidades queda manifiesta a través del análisis de la movilidad relativa entre las clases I, II y VI, VII. Por ejemplo, los hombres provenientes de la clase II tienen, aproximadamente, 18 veces más probabilidades que los hombres provenientes de la clase VI de encontrarse en la clase II en vez de la clase VI. Esto es bastante contundente, pero menos desigual que el hecho que los hombres provenientes de la clase I tienen 72 veces más probabilidades (que los hombres provenientes de la clase VII) de encontrarse en la clase I en vez de la clase VII. Asimismo, la desigualdad de oportunidades entre las clases superior y rural es muy dramática. Sin embargo, hay que tomar estas tasas con cautela debido a las características de la muestra (pequeña cantidad de casos de los destinos rurales). No obstante, creo que la desigualdad será aún mayor si incluimos a la población rural. Aquí estamos considerando lo que podría considerarse el segmento más exitoso de la población rural: la población que trabaja cerca a las ciudades.

Los profesionales y gerentes de los niveles superior e inferior tienen distintos patrones de movilidad. De acuerdo con estos resultados, por ejemplo, las probabilidades que tienen los hombres provenientes de la clase I de terminar en la clase I en vez de la clase II son apenas 1,44 veces mayores que para los hombres que tienen padres en la clase II. Al mismo tiempo, si comparamos las probabilidades en las hileras horizontales 1 y 2 , es posible observar cómo difieren las posibilidades relativas para los profesionales y gerentes de los niveles superior e inferior. Por ejemplo, como ya se indicó, las 
probabilidades que tienen los hombres provenientes de la clase I de encontrarse en la clase I en vez de la clase VII son 72 veces mayores que la probabilidades que tienen los hombres provenientes de la clase VII de encontrarse en la clase I en vez de la clase VII, mientras que los hombres provenientes de la clase II tienen una probabilidad 13 veces mayor. Este es indicativo de los problemas que tienen algunos de los ocupantes de la clase profesional de nivel inferior para mantener su condición social dentro de la estructura de clases.

La igualdad de oportunidades (las celdas donde la tasa de probabilidad es igual o próxima a 1) está presente en algunas zonas de la estructura de clases; por ejemplo, en los movimientos entre las clases IIIa y IIIb, las clases II y IV, las clases II y V, IIIbVI y IIIb-VII. Tal como se planteó en las hipótesis, se descubre importantes patrones de fluidez en los niveles intermedios, en los niveles inferiores y entre la clase IIIb y niveles inferiores.

En general, la desigualdad de oportunidades sigue siendo muy importante en la estructura social peruana, especialmente entre las clases superiores e inferiores. Este patrón coincide más con una sociedad que se aparta dramáticamente del ideal de una genuina apertura. Es interesante contrastar estos resultados con el que obtuvimos de las tasas absolutas en las cuales se mostraban patrones ascendentes de movilidad.

Es cierto que la estructura de clases peruana se ha vuelto menos homogénea en su composición y una buena cantidad de individuos se ha desplazado de ocupaciones en niveles inferiores a otras en niveles superiores (aproximadamente, $40 \%$ de la tasa de movilidad total), pero eso no significa, necesariamente, que se haya logrado una mayor igualdad de oportunidades. Por el contrario, la sociedad peruana (como la mayoría de las sociedades) aún está lejos de alcanzar esa meta. También es interesante contrastar estas barreras a la movilidad de largo alcance con la fluidez que se ha encontrado en los niveles intermedios, donde se presentan menos dificultades para los diversos procesos de movilidad social. Lo mismo se puede decir de la recomposición que ha experimentado esa franja intermedia, debido no sólo a la movilidad ascendente de los trabajadores, sino también debido a la movilidad descendente de los profesionales tipo II. A ello contribuye también la desaparición de lo que antes era una "alianza" entre los empleados y los sectores altos de la sociedad peruana. Los primeros están ahora más cercanos a los grupos bajos que a los anteriores.

En general, se puede decir que el patrón de movilidad en la sociedad peruana está mayormente impulsado por esta fluidez intermedia más que por una movilidad de largo alcance. Las franjas intermedias se han reconstituido notablemente en el Perú, al mismo tiempo que los extremos configuran estamentos distantes. La reducción de las brechas entre unos y otros aún es un objetivo no alcanzado. En ese sentido, los cambios estructurales que hemos experimentado se han localizado más en lo que podríamos llamar un "ensanchamiento" de las clases medias, las cuales se componen ahora de sectores cuyos orígenes se ubican tanto en la mismos grupos intermedios, como en los sectores bajos y en los sectores altos que experimentaron movilidades descendentes. 


\section{CONCLUSIONES}

Este estudio confirma los límites que los procesos de modernización tienen para alterar la igualdad de oportunidades cuando los factores exógenos como el crecimiento económico no se mantienen constantes o cuando las propias relaciones sociales y culturales no cambian significativamente. En el Perú procesos importantes de movilidad social y por lo tanto de reconstitución de nuestra estructura social han sido experimentados, al mismo tiempo que la polarización o las distancias relativas entre extremos se han mantenido. Esto define una estructura social bastante compleja, muy dinámica entre las zonas intermedias y bajas, pero casi estamental entre las zonas altas y bajas. Los cambios que ocurren y transforman las dinámicas de los sectores intermedios y bajos no afectan las diferencias estructurales que se ligan más a la reproducción de diferencias culturales entre clases altas y bajas.

¿Qué tan diferentes somos de otras sociedades? Dicho patrón puede ser comparado con otros donde existen también importantes procesos de movilidad relativa en las zonas intermedias pero donde las diferencias entre los extremos son menos acentuadas tales como los casos de Israel (Yaish, 2001) y Polonia (Erikson \& Goldthorpe, 1992). En ambos casos tanto por el impacto de los procesos migratorios como por los efectos de la revolución comunista, la movilidad de largo aliento no es tan pronunciada. Nuestro caso se asemeja más al de Hong Kong (Chan et al., 1994) donde las diferencias lingüísticas entre clases altas y bajas no han permitido una mayor apertura social y las distancias sociales se han mantenido al mismo tiempo que se dio mucha movilidad en zonas intermedias (Benavides, 2002).

\section{Referencias citadas}

BENAVIDES, M., 2002 - Class Mobility and Equality of Opportunities in the Context of Erratic Modernization: The Peruvian case. Ph.D. Dissertation. Pennsylvania State University.

BECKER, D., 1983 - The New Bourgeoisie and the Limits of Dependency: Mining, Class, and Power in Revolutionary Peru, 419p.; Princeton: Princeton University Press.

CHAN, T.W., LUIS, T.L. \& WONG, T.W., 1994 - A comparative analysis of social mobility in Hong Kong. European Sociological Review, 11: 135-155.

CASTELLS, M. \& PORTES, A., 1989 - The Informal Economy: Studies in Advanced and Less Developed Countries, 327p.; Baltimore: John Hopkins University Press.

CHAPLIN, D., 1967 - The Peruvian Industrial Labor Force, 324p.; Princeton, NJ.: Princeton University Press.

CHAPLIN, D., 1969 - Peruvian stratification and mobility. Revolutionary and developmental potential. In: Structured Social Inequality (C. S. Heller ed.); London: MacMillan.

ERIKSON, R. \& GOLDTHORPE, J.H., 1992 - The Constant Flux. A Study of Class Mobility in Industrial Societies, 429p.; Oxford: Clarenton Press.

FRANCO, C., 1991 - Exploraciones en "otra modernidad": de la migración a la plebe urbana. In: La Otra Modernidad, Imágenes de la Sociedad Peruana; Lima: CEDEP.

GERMANI, G., 1981 - The Sociology of Modernization, 266p.; New Brunswick and London: Transaction Books. 
GOLDTHORPE, J. H., 1987 - Social Mobility and Class Structure in Modern Britain, 377p.; Oxford: Clarendon Press.

GOLDTHORPE, J.H., 2000 - On Sociology. Numbers, Narratives and the Integration of Research and Theory, 337p.; Oxford: Oxford University Press.

GUADALUPE, C., 1991 - De Levitan a Remora. Debates en Sociología, 16: ; Lima: Universidad Catolica del Perú.

HAUSER, R., 1978 - A structural model of the mobility table. Social Forces, 56, 3: 919-953.

HOUT, M., 1983 - Mobility Tables, 93p.; Beverly Hills: Sage.

KELLEY, J. \& KLEIN, H., 1981 - Revolution and the Rebirth of Inequality: A Theory Applied to the National Revolution in Bolivia, 279p.; University of California Press.

LIPSET, S. M. \& ZETTERBERG, H. L., 1959 - Social mobility in industrial society. In: Social Mobility in Industrial Society ( S. M. Lipset \& R. Bendix Eds.), 309p.; Berkeley: University of California Press.

NUGENT, G., 1992 - El laberinto de la choledad, 140p.; Lima: Fundacion Ebert.

PARKER, D.S., 1998 - The Idea of Middle Class: White Collar Workers and Peruvian Society, 1900-1950, 266p.; University Park: The Pennsylvania State University Press.

PÁSARA, L., DELPINO, N., VALDEAVELLANO, R. \& ZARZAR, A., 1991 - La otra cara de la luna. Nuevos actores sociales en el Perú, 208p.; Lima: CEDYS.

RAWLS, J., 1971 - A theory oj justice, 607p.; Cambridge: Mass.

ROEMER, J., 1998 - Equality of oportunity, 120p.; Cambridge, Massachussetts: Harvard University Press.

RONA-TAS, A., 1994 - The first shall be last? Entrepreneurship and communist cadres in the transition for socialism. American Journal of Sociology, 100: 40-69.

SOTO de, H., GERSHI, E., GHIBELLINI, M. \& EL ILD (en colaboración con), 1986 - El otro sendero. La revolución informal, 317p.; Lima: Editorial El Barranco.

TORERO, M., SAAVEDRA, J., NOPO, H. \& ESCOBAL, J., 2002 - The economics of social exclusion in Peru: An invisible wall?; Lima: GRADE.

YAISH, M., 2001 - Class structure in a deeply divided society: Class and ethnic inequality in Israel, 1974-1991. British Journal of Sociology, 52(3): 409-439. 